\title{
Estado de Salud Oral de una Muestra de Enfermos con COVID 19 del Hospital Dr. Sótero del Río, Santiago de Chile
}

\author{
Oral Health Status of a Sample of COVID 19 patients from \\ the Dr. Sótero del Río Hospital, Santiago de Chile
}

\author{
Verónica Palacios Inostroza ${ }^{1,2}$; Carla Squella Jara ${ }^{3}$ \& Fernanda Dowling Schilling ${ }^{4}$
}

\begin{abstract}
PALACIOS, I. V.; SQUELLA, J. C. \& DOWLING, S. F. Estado de salud oral de una muestra de enfermos con COVID 19 del Hospital Dr. Sótero del Río, Santiago de Chile. Int. J. Odontostomat. 15(4):1026-1034, 2021.

RESUMEN: Con el objetivo de conocer la presencia de manifestaciones clínicas específicas de COVID-19 en la cavidad oral y describir el estado de salud oral de los enfermos se realizó un examen de la cavidad oral básico a 99 personas con COVID-19 internados en la Unidad de Paciente Crítico (UPC) y en otras dependencias del Hospital Dr. Sótero del Río en Santiago de Chile, durante julio 2020. Del total de enfermos 51 estaban en estado crítico con ventilación mecánica invasiva (VMI), edad promedio 55 años y 48 se encontraban sin VMI, con 66 años en promedio, la mayoría era de sexo masculino, con $25,5 \%$ de mujeres con VMI y $37 \%$ entre los sin VMI, la Hipertensión arterial fue la co- morbilidad sistémica más frecuente entre los enfermos seguida por Diabetes mellitus y Obesidad tanto entre los en VMI como sin ella. Respecto a salud oral se observó un predominio de lesiones ulcerativas y erosivas en 57 \% de las personas en VMI, el número de estas y su patrón clínico sin embargo pareció inespecífico, también destacó la alta frecuencia de sequedad de la cavidad oral, alcanzando un 54 $\%$ en los enfermos en estado crítico y un 34 \% en los de menor gravedad, llamó la atención la palidez presente en las mucosas y el aspecto clínico de la lengua. La presencia de Inflamación periodontal se pudo evidenciar clínicamente, pero no fue posible determinar el nivel de inserción, ni evaluar con radiografías a los enfermos, siendo solo un dato aproximado. Los dientes se encontraban en general en regular estado concordante a lo que se observa en grupos poblacionales similares en Chile. No se pudo determinar la presencia de lesiones específicas de COVID-19 en este grupo estudiado.
\end{abstract}

PALABRAS CLAVE: COVID-19, manifestaciones orales.

\section{INTRODUCCIÓN}

Todo parte cerca del año nuevo en Wuhan, diciembre 2019, cuando surge un nuevo patógeno que el comité Internacional de taxonomía de Virus denominaría como SARS-CoV-2 (severe acute respiratory syndrome-coronavirus 2) agente etiológico de COVID19 , que nos llevó a una Pandemia histórica que ha cobrado millones de vidas, marcando un hito para la humanidad. El 30 de enero 2020, la Organización Mundial de la Salud (OMS) lo denominó una Emergencia de Salud Pública de importancia Internacional (ESPII) y el 1 de abril del mismo año se declara Pandemia. En junio de 2021 a nivel internacional se han reportado un total de176.945.596 casos confirmados y 3.836 .828 fallecidos por COVID-19 (OMS,2021) y en Chile para igual fecha hay 1.800 .673 casos acumulados de COVID-19, con 40.365 personas fallecidas que incluyen los confirmados y probables. (Informe Epidemiológico 130) (Ministerio de Salud, 2020). La ciencia ha trabajado intensamente, se conoce la presentación clínica sistémica de COVID-19, se avanza en el manejo clínico de la enfermedad, sin encontrar aún un tratamiento curativo y se han desarrollado vacunas que buscan la inmunidad de rebaño. Ya no es una novedad la manera en que ingresa el virus al hos-

\footnotetext{
${ }^{1}$ Especialista en Patología Buco Máxilo facial, Hospital Dr. Sótero del Río, Santiago, Chile.

2 Escuela de Odontología Pontificia Universidad Católica de Chile, Santiago, Chile.

${ }^{3}$ Especialista en Endodoncia, Hospital Dr. Sótero del Río, Santiago, Chile.

${ }^{4}$ Especialista en Rehabilitación, Hospital Dr. Sótero del Río, Santiago de Chile.
} 
pedero humano a través de su proteína Spike que interactúa con receptores ACE 2 facilitado por el clivaje de los mismos que realiza una serina proteasa transmembrana identificada como TMPRSS2 (Hoffmann et al., 2020), estos receptores ACE 2 están presentes en varios tejidos,también en los queratinocitos de la mucosa oral, especialmente en la superficie de la lengua,esto permitiría explicar la disfunción gustativa,que un porcentaje importante de los enfermos relata y que podría ser el resultado del efecto citopático del virus, también son expresados en glándulas salivales que pueden actuar como reservorio y justifican parte del mecanismo de transmisión del virus a través de la saliva (Patel et al., 2020; Machhi et al., 2020; Amorim Dos Santos et al., 2021), el virus se ha encontrado incluso en Fluido crevicular (Gupta et al., 2021) pero a pesar de ello lo que ocurre en cavidad oral aún no se ha esclarecido, numerosos investigadores han hecho reportes (Cruz Tapia et al., 2020; Sinadinos \& Shelswell, 2020; Amorim dos Santos et al.; Brandão et al., 2021; Soares et al., 2021) pero nada concluyente, entonces con la intención de aportar al conocimiento de la enfermedad el Servicio de salud Metropolitano Sur Oriente (SSMSO), Hospital Dr. Sótero del Río se motivó a escudriñar en este tema.

COVID-19 es una infección respiratoria multisistémica que se puede manifestar con fiebre, tos seca, mialgias, disgeusia, disosmia entre otras, el compromiso respiratorio puede ser una Neumonía que progrese a Síndrome Respiratorio Agudo Severo (Young et al. 2020),con Síndrome de Respuesta Inflamatoria Sistémica (SIRS) desencadenado por una verdadera tormenta de citoquinas capaces de conducir a Falla orgánica Múltiple (FOM) que ha sido causa de muerte de nuestros enfermos, con mayor riesgo para quienes padecen enfermedades sistémicas, como HTA, Diabetes mal controlada (Mazucanti \& Egan, 2020), Obesidad, inmunodepresión o envejecimiento que hace al ser humano más proclive a sufrir cuadros respiratorios graves. La vulnerabilidad a las infecciones en general de los Diabéticos con un control metabólico inadecuado es conocida, ejemplos documentados son la relación de Diabetes con Periodontitis Crónica (Baeza et al., 2020) y con Mucormicosis (Hongo Negro) (Bonifaz et al., 2021) entonces sin duda afectaría la evolución de la infección por SARS-CoV-2,surgiendo interrogantes más puntuales como el vínculo entre COVID-19 y Periodontitis Crónica (Coke et al., 2021), que hacen necesario revisar cautelosamente los datos publicados para obtener conclusiones acertadas (Marouf et al., 2021). La Diabetes mellitus, predispo- ne o agrava la patología coronaria y si va de la mano de HTA y Obesidad sin duda que la mortalidad se incrementa, por eso son quienes han tenido los peores resultados enfrentando la Pandemia causada por COVID-19.

Lo que la comunidad e incluso los profesionales de la salud que no son intensivistas tal vez desconozcan, es lo complejo del tratamiento de enfermos con COVID-19 si requieren una terapia en pronación o con ventilación mecánica invasiva debiendo ser intubados para mejorar su función respiratoria. En este escenario, el enfermo grave debe ser sedado, polimedicado y depende absolutamente de la terapia intensiva para salvar su vida (Machhi et al.), es aquí donde son múltiples los aspectos que el equipo de salud vigila para lograr el éxito,aquí son héroes reales quienes cuidan a estos enfermos, arriesgan su vida, su capacidad intelectual, fuerza espiritual y física no es una metáfora:" el enfermo que ingresa a cuidados intensivos está desamparado, muchas veces solo, ve irse sus sueños, necesita de fraternidad, se enfrenta a la muerte (Hernández Poblete, 2000).

\section{MATERIAL Y MÉTODO}

Se realizó un estudio observacional descriptivo del estado de salud oral de 99 enfermos con infección por SARS -CoV-2, COVID-19, confirmados con PCRTR (reacción en cadena de polimerasa-transcriptasa reversa) internados en el hospital Dr. Sótero del Río,en la Unidad de paciente crítico (UPC) con Ventilación Mecánica Invasiva(VMI) o en unidades para enfermos menos graves, a quienes se realizó un examen clínico básico de salud oral, incluyendo mucosas, labios, piel peri oral y aspecto general de los dientes presentes en boca, esto se realizó ocupando elementos de Protección personal según protocolo e instrumental básico, siendo asesorados por los profesionales a cargo de los enfermos, contando con la autorización del comité ético científico del SSMSO, se obtuvo acceso a registro de información clínica respecto al mismo tópico de la salud oral y comorbilidades. Por el complejo estado de los enfermos toda la evaluación se hizo estando en su cama y solo se registró fotográficamente casos muy puntuales con consentimiento de los enfermos, sus tutores o médico responsable ajustados a las normas éticas y de confidencialidad. La información obtenida se presenta dando a conocer la condición de salud oral general de esta muestra de personas con COVID-19 hospitalizadas en esta institución. 


\section{RESULTADOS}

Se examinó a 99 personas adultas, en julio de 2020, 51 estaban en la UPCconectados a VMI y 48 en unidades de cuidados intermedios, sin VMI, la gravedad de quienes estaban en la UPC, se expresa en parámetros clínicos y exámenes de laboratorio, pero como indicador del compromiso sistémico para este estudio solo se registró el valor de Proteína $\mathrm{C}$ reactiva en los más graves, encontrándose un promedio de 221 $\mathrm{mg} / \mathrm{dl}$ para los pacientes en VMI (rango de normalidad es inferior a $5 \mathrm{mg} / \mathrm{dl}$ ), valor considerado tan alto como lo registrado por otros trabajos (Marouf et al.).

Los pacientes en UPC eran de un promedio de 55 años, similar al estudio de Nanshan de Wuhan en 2020, (con un rango entre 18 y 74 años) y aquellos no UPC, menos graves, 66 años promedio (rango entre 20 y 91 años ), es decir los más graves tenían un promedio de edad 11 años menor, siendo en ambos grupos la mayoría hombres con solo un $25,5 \%$ de mujeres en el grupo con VMI y un $37 \%$ de ellas en el grupo menos grave.

Respecto a las morbilidades de estos enfermos, hay un predominio de Hipertensión arterial en $56 \%$ de enfermos en UPC(VMI) y $64 \%$ entre los menos graves, seguido por Diabetes mellitus en los dos grupos, alcanzando un $42 \%$ en los en VMl y $39 \%$ en los otros, la obesidad se registra en $23 \%$ en los enfermos en UPC y un $10 \%$ en los que no se encontraban en UPC tal como la literatura lo señala y se confirma en los más graves (Tabla I).

Tabla I. Registro de Co morbilidades en los dos grupos de enfermos COVID-19.

\begin{tabular}{lcc}
\hline COMORBILIDADES & $\begin{array}{l}\text { Enfermos en } \\
\text { UPC }\end{array}$ & $\begin{array}{l}\text { Enfermos no } \\
\text { UPC }\end{array}$ \\
\hline Hipertensión arterial & $56 \%$ & $64 \%$ \\
Diabetes mellitus & $42 \%$ & $39 \%$ \\
Obesidad & $23 \%$ & $10 \%$ \\
Enf. cardiovasculares & $17 \%$ & $20 \%$ \\
Enf. Renal Crónica & $5.9 \%$ & $14.5 \%$ \\
Trasplantados & $7.8 \%$ & 0 \\
Daño Hepático Crónico & $2 \%$ & $4 \%$ \\
Apnea Obstructiva del & $3.9 \%$ & 0 \\
Sueño & & \\
\hline
\end{tabular}

Respecto a las alteraciones presentes en la cavidad oral (Fig. 1) lo más común es encontrar lesiones ulcerativas y erosivas en los pacientes que se encuentran conectados a VMI, estando presentes en un

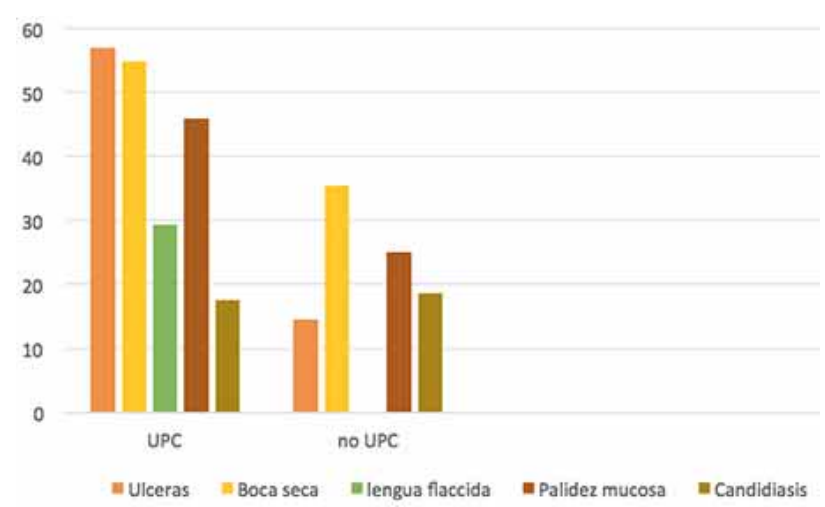

Fig. 1. Porcentaje de enfermos con Covid 19 con alteraciones orales.

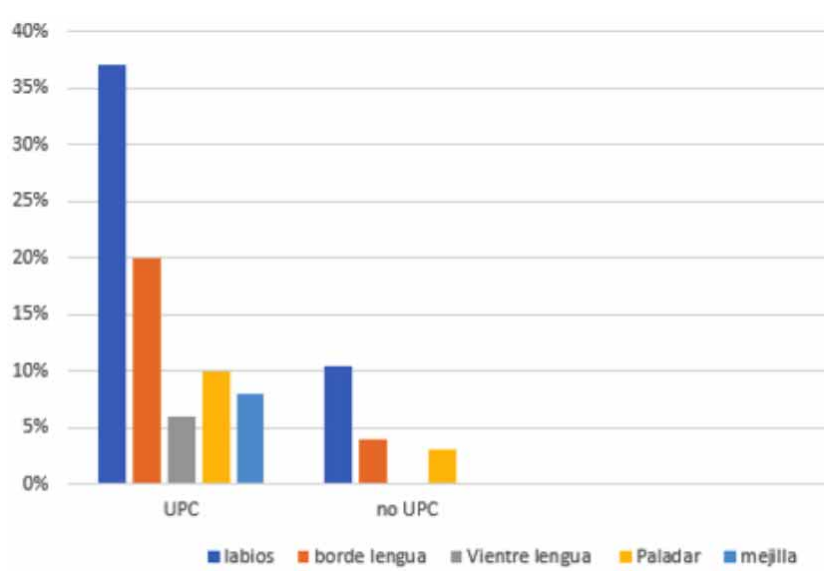

Fig. 2. Distribución de lesiones ulcerativas.

$57 \%$ de las personas de este grupo,en contraste con sólo un $14,5 \%$ de quienes no estaban sometidos a ventilación mecánica, su presentación clínica y distribución es variable predominando en labios, márgenes y vientre de lengua, debemos aclarar que se registró el porcentaje de enfermos con ulceras o erosiones, pero hay que destacar que muchos de ellos presentaban múltiples lesiones con distribución (Fig. 2) forma y tamaño variable, tipo aftas, otras tipo herpes, pero sin todos los criterios para precisar el diagnóstico clínico. Es importante señalar que de los 99 pacientes examinados son pocos los que presentan lesiones ulcerativas $u$ otras que se puedan atribuir a la expresión de una enfermedad específica, por ejemplo 2 enfermos presentaron hallazgos que se parecen clínicamente a Eritema Multiforme, pero esto es discutible porque además del compromiso oral no tenían afectadas otras mucosas y su rango de edad era superior a lo que se describe para esta condición (Fig. 3). Otros dos pacientes graves presentaban lesiones ulcerativas con forma y distribución eventualmente 

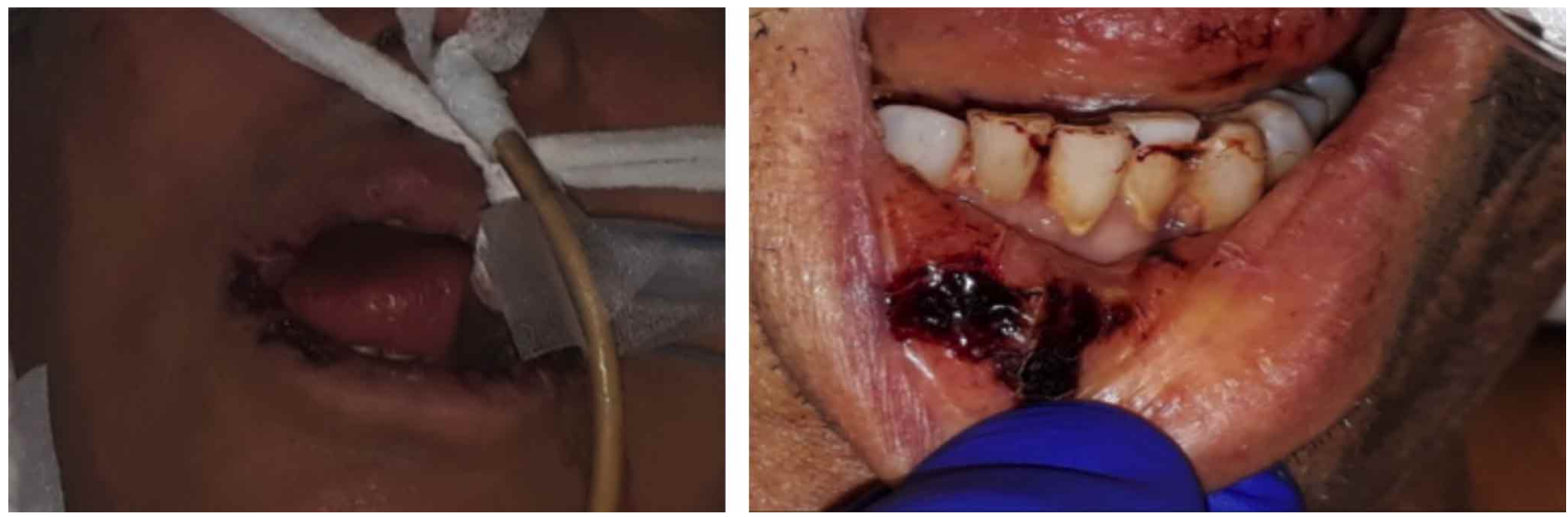

Fig. 3. muestran lesiones ulcerativas necrótico-hemorrágicas.
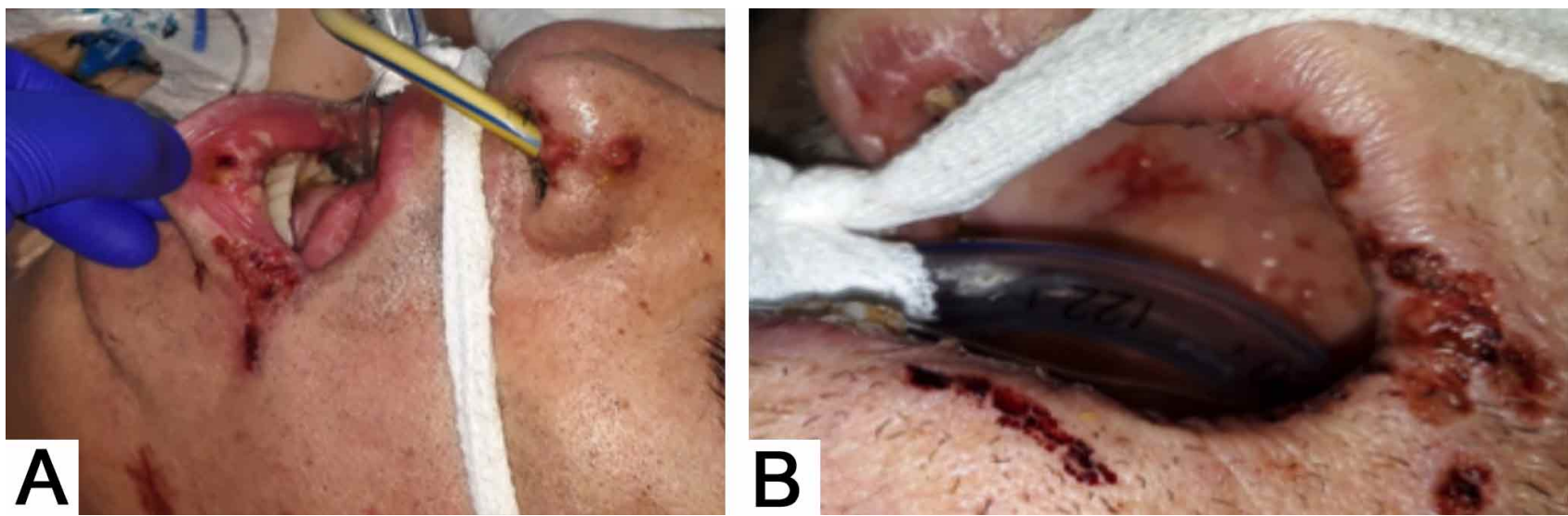

Fig. 4 Lesiones ulcerativas múltiples e irregulares con un patrón herpetiforme.

asociadas a reactivación intraoral y peri oral de Virus Herpes Simple tipo 1, situación que no se pudo confirmar con exámenes de laboratorio (Figs. 4a y b).

La sequedad de la cavidad oral se destacaba a la inspección visual de forma evidente y dramática en un $54,9 \%$ de los enfermos en UPC y en $35,4 \%$ de los no UPC, pero no se realizó evaluación de flujo salival por el complejo estado de las personas, sin embargo, cualitativamente se pudo ver la disminución, consistencia y las consecuencias de su escases especialmente en lengua y labios.

Se observó una marcada palidez de las superficies mucosas de lengua, encías y piso de la cavidad oral en todos los grupos, presente en $46 \%$ de los enfermos más graves en UPC y $25 \%$ en los no UPC. Otra observación en las personas más graves en VMI es el aspecto relajado, fláccido, agrandado o edematoso de la lengua, lo cual se destacó en el 29,4 $\%$ de ellas, hallazgos perceptibles a la palpación e inspección y ausente en los enfermos que no se encuentran en VMI.
Con relación al estado de los dientes (Tabla II) su situación no difiere de la realidad nacional para estos rangos de edad, entonces en los adultos mayores predominaban su ausencia parcial o total y en los más jóvenes, estos dientes estaban presentes, aunque ningún enfermo tenía dentición completa, además es necesario comentar que en un $27,4 \%$ de las personas estos se encontraban, con irregularidades o márgenes cortantes (Tabla II).

Tabla II. Estado Clínico general de dientes y Periodonto.

\begin{tabular}{lcl}
\hline Condición clínica & $\begin{array}{l}\text { Enfermos } \\
\text { UPC }\end{array}$ & $\begin{array}{l}\text { Enfermos } \\
\text { no UPC }\end{array}$ \\
\hline Inflamación periodontal & $43 \%$ & No \\
& & evaluado \\
Dientes con bordes cortantes & $27,4 \%$ & $8,3 \%$ \\
Ausencia Total de dientes & 0 & $25 \%$ \\
Desdentado Tot Sup / Parc Inf & 0 & $14 ., 5 \%$ \\
Desdentado Parcial Sup. e Inf. & $100 \%$ & $58 \%$ \\
Rehabilitación con implantes & 0 & $2 \%$ \\
\hline
\end{tabular}

La presencia de inflamación periodontal solo se pudo evaluar en forma general, sin posibilidad de determinar la pérdida de inserción, ni examen 


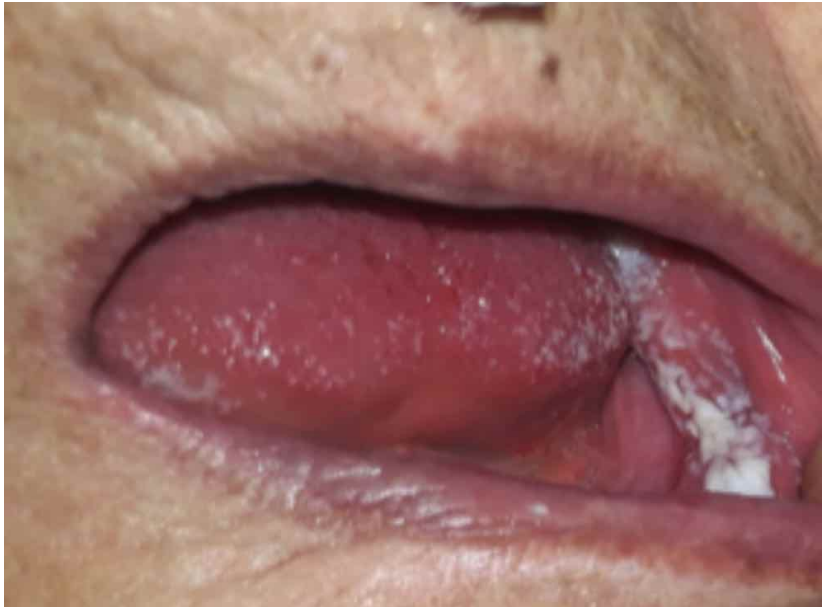

Fig. 5 Lesiones de Candidiasis.

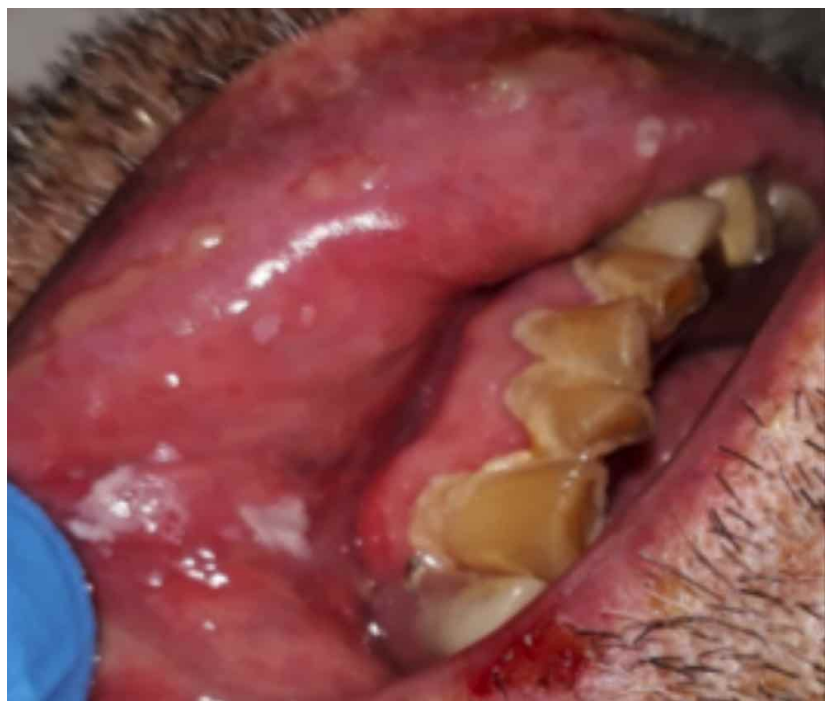

Fig. 6 Lesiones Ulcerativas.

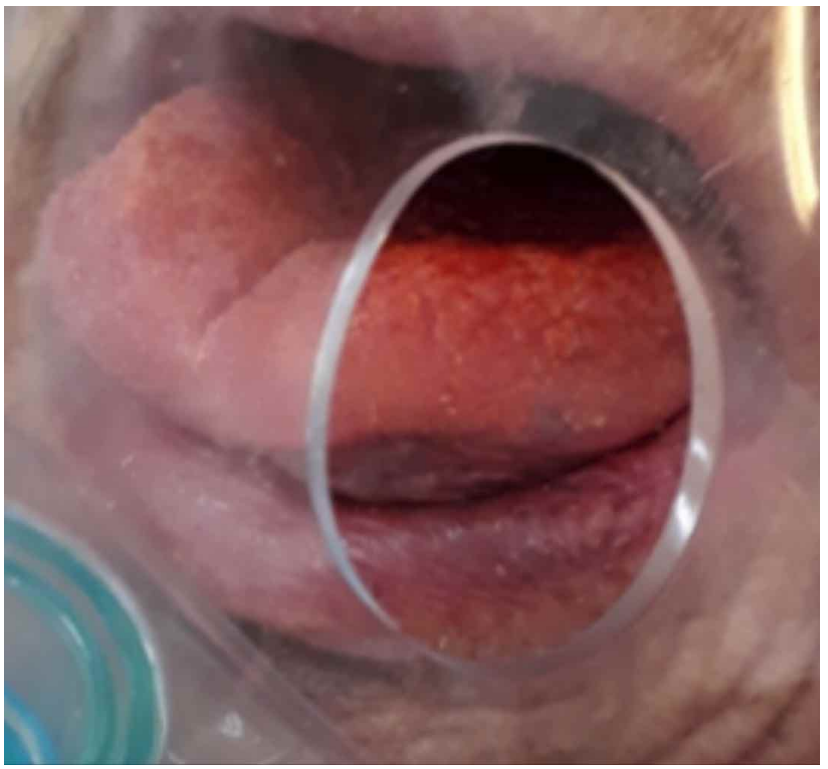

Fig. 7 Sequedad oral intensa.

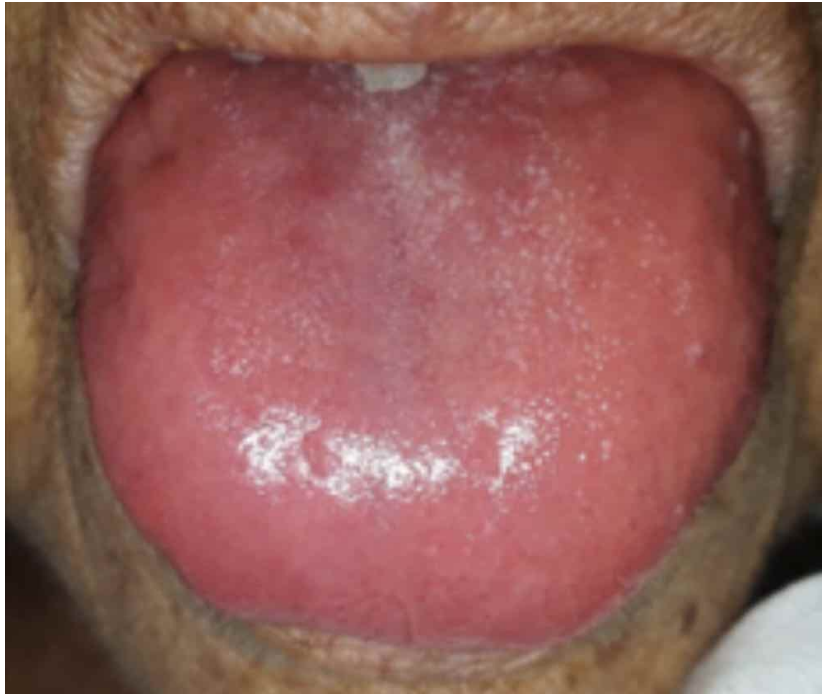

Fig. 8 Lengua, lisa, pálida y seca.

radiográfico, por las dificultades propias de la condición de salud de los enfermos, estimándose su presencia en $43 \%$ de los enfermos de UPC basándose en la inflamación clínica asociada a depósitos de cálculo y placa bacteriana (Tabla II) (Patel et al.).

Otro hallazgo que se pudo evidenciar es la presencia de Candidiasis, infección oportunista clínicamente evidente con una frecuencia similar en los enfermos más graves y entre los menos graves que tienen además mayor edad, presente en $17,6 \%$ y $18,6 \%$ respectivamente (Fig. 5).

\section{DISCUSIÓN}

Las características del grupo de pacientes en cuanto a edad y sexo coinciden con los primeros reportes publicados desde la ciudad de Wuhan (Nanshan et al. 2020, Wang et al., 2020). Actualmente (año 2021) los enfermos que ingresan a las unidades de cuidados intensivos en Chile son de menor edad, fenómeno explicado probablemente por la vacunación masiva que se está llevando a cabo en nuestro país que ha beneficiado inicialmente a la población de más edad.

Enfatizamos que en nuestro estudio se hizo un examen clínico en un momento determinado de la estadía hospitalaria de los enfermos y además se revisó las historias clínicas para valorar antecedentes que estuvieran descritos en estas en el momento preciso del ingreso de los enfermos o en los días previo a nues- 
tro examen, sin encontrar registros de lesiones .Una de las manifestaciones orales más prevalentes en pacientes COVID-19 publicada son las alteraciones gustativas presentes en promedio de $45 \%$ de los enfermos (Amorim Dos Santos et al.), pero en este estudio no se pudo evaluar por las características de ellos, centrándonos en las alteraciones objetivas que pudimos observar. Si se analizan los hallazgos presentes en estos enfermos con COVID-19 sintomáticos confirmados con PCR TR y se contrastan con aquello que los especialistas han tratado de documentar podemos discutir que si bien las lesiones ulcerativas son comunes en enfermos con COVID-19 (Iranmanesh et al., 2021; Egido-Moreno et al., 2021), no se vio que tuvieran un patrón determinado ni específico, creemos que pueden tener explicaciones variables y en general pudieran no ser efecto directo del patógeno (SARS -CoV-2), su ubicación coincide con áreas de mayor presión o roce de dispositivos que mantienen con vida a estos enfermos o en cercanía de dientes en regular estado que podrían ocasionar traumatismos sobre la lengua o labios, que son las áreas más afectadas por ulceraciones en este grupo de personas, considerando que durante los episodios de Pronación o en VMI toda la musculatura está relajada farmacológicamente, eso justifica porque la lengua se vea agrandada, edematosa e hipo reactiva, más vulnerable nos parece a traumatismos, por otra parte los enfermos en decúbito supino suelen estar con la boca abierta por la sedación profunda, sin deglutir, a lo que se suma un gran resecamiento oral (Figs. 7 y 8) por evaporación o por deshidratación, en algunos casos producto de su estado de salud grave (fiebre) o como efecto de medicamentos que pueden causar hipo salivación, dado que la sequedad oral se aprecia en $54,9 \%$ de los enfermos en VMI, de Biadsee et al. (2020), describe un alto porcentaje de sequedad de la cavida oral, a pesar de que en su estudio no son enfermos hospitalizados. Si analizamos porqué la mucosa oral en un enfermo grave, es tan frágil podemos decir que precisamente la falta de saliva y sus consecuencias en cuanto a lubricación y protección inmune,pueden ser parte de la respuesta y además la reducción de flujo sanguíneo en la mucosa por el ajuste hemodinámico sistémico que busca proteger territorios más sensibles a la hipoxia, dejando la mucosa oral mal perfundida (Hernández et al.. 2013, 2018), esto es coherente con el signo de llene capilar alterado en enfermos graves, entonces cobra valor las cifras de palidez registradas en nuestro trabajo que podrían ser manifestación de aquello, alterando esta barrera de protección mucosa ante el roce, la presión mecá- nica o facilitando tal vez el ingreso de flora microbiana en un ambiente de disbiosis (Hoque et al., 2021) que probablemente está presente en la boca como consecuencia del uso de potentes antimicrobianos para tratar Infecciones asociadas a la atención de salud (IAAS)o reagudizaciones de cuadros crónicos, en este estudio un $76 \%$ de los enfermos más graves requirió antibióticos en algún momento de su estadía, similar a lo descrito en otros trabajos referidos a este tema de los cuidados intensivos.Acá es necesario señalar que la identificación de bacterias con técnica corriente se ve dificultada por este antecedente y se recomienda el uso de técnicas metagenómicas (Cox et al., 2020) lo cual complejiza aún más la situación.

Los enfermos en VMI de este grupo reciben, con una vocación admirable, por protocolo cada 6 horas, aseo de la cavidad oral que incluye irrigación con clorhexidina al $0,12 \%$ y aspiración, lo cual sin duda disminuye la flora microbiana en su cavidad oral con el objetivo de disminuir la posibilidad de neumonías por aspiración (Álvarez Lerma, 2014) hoy se estudia si las bacterias de la cavidad oral pueden tener participación en las complicaciones potenciales de estos enfermos con COVID-19 (Patel et al.), teniendo presente que quienes ingresan a cuidados intensivos y son sometidos VMI pueden tener complicaciones infecciosas procedentes de condiciones odontoestomatológicas crónicas previas que se reagudizan durante su hospitalización.

Se discute si las ulceraciones observadas siguen un patrón tipo aftas o como ulceras necrotizantes (Brandão et al.) (Figs. 6) o si se parecen a lesiones herpéticas de las formas de herpes simple (Tang et al., 2020; La Rosa et al., 2021) o zoster y se concluye que solo en un par de casos en enfermos muy graves podemos sospechar que lo registrado se ajusta al criterio clínicos de reactivación de Herpes simple (Figs. 4 A-B) y otro par de personas presenta lesiones sugerentes de Eritema multiforme por el tipo de compromiso labial pero es discutible porque carecen de otros componentes del cuadro clínico (Jimenez-Cauhe et al., 2020). Por otra parte hay descritos en la literatura casos clínicos estudiados además con histopatología donde se propone que los enfermos con COVID-19 pueden tener manifestaciones de trombosis y vasculitis en la mucosa oral (Cruz Tapia et al.; Soares et al.), en los enfermos de nuestro grupo no apreciamos lesiones clínicas sugerentes de esto, observando algunas equimosis en distintas ubicaciones pero se podían interpretar como consecuencia de que estos enfermos suelen estar con anticoagulantes como heparina de 
bajo peso molecular y algunos presentan trombocitopenia como expresión de su condición.

Luego del análisis de la información obtenida, consideramos que probablemente los hallazgos clínicos de estos enfermos no permiten afirmar que existen lesiones específicas de COVID-19 en la mucosa oral, en este grupo estudiado, si podríamos proponer que hay condiciones que acompañan de forma significativa a enfermos graves(VMI en UPC) y que pueden ser consecuencia de su fragilidad, del compromiso sistémico,labilidad inmunológica, morbilidades pre existentes o factores locales previos o inherentes a su condición de gravedad.

Respecto a la presencia de dientes en los grupos de más edad se aprecia algo similar a lo descrito en la encuesta Nacional de Salud (Ministerio de Salud, 2018) a medida que los enfermos tiene más edad aumenta el porcentaje de dientes perdidos y de dentición no funcional, esto se ve reflejado en lo que observamos en este grupo de personas en quienes no fue posible realizar un examen periodontal adecuado a los estándares mínimos por lo que solo reportamos que de acuerdo a la visualización clínica de signos inflamatorios, presencia de depósitos de placa bacteriana y cálculo es posible comentar que la inflamación periodontal está presente en un $43 \%$ de los enfermos conectados a VMI, que tiene un promedio de edad de 55 años, refleja probablemente lo que se describe en grupos etarios similares a la población examinada y asociado a los determinantes sociales de nuestros pacientes. Lo publicado por el equipo de Mariano Sanz (Marouf et al.) respecto a que la Periodontitis Crónica aumenta el riesgo de morir de los enfermos de COVID-19, no debe sorprender porque ellos tienen una flora periodonto patógena que los pone en riesgo y el solo hecho de tener esta Periodontitis crónica da cuenta que han sido más vulnerables inmunológicamente, lo mismo que ya sabemos lo es un Diabético mal controlado o un inmunocomprometido.

\section{CONCLUSIÓN}

En este estudio observacional y descriptivo, se ve que prevalecen las lesiones ulcerativas y erosivas en la cavidad oral de los enfermos hospitalizados con COVID-19, sin embargo,su número, distribución y aspecto clínico parece inespecífico y reflejarían el estado crítico de los enfermos no compromiso Oral de la infección por SARS-CoV-2.
La sequedad oral en los enfermos con COVID19 tanto los más graves y conectados a VMI, como en menor proporción los menos graves, puede ser en parte una consecuencia de la Infección con SARS-CoV-2 si consideramos que el virus compromete las glándulas salivales, como lo han descrito numerosos autores (Hoffmann et al.; Soares et al.) obedece también a la grave condición de salud, a los fármacos usados, incluso a las morbilidades previas, de acuerdo a esto recomendamos siempre lubricar y proteger la mucosa.

La lengua se encuentra muy relajada e hiporeactiva, probablemente más susceptible a traumas, por lo tanto en los enfermos graves debe ser protegida, también considerar la hipoperfusión periférica visualizada en la mucosa oral empalidecida nos debe hacer comprender la vulnerabilidad de los enfermos en estado crítico tanto a las ulceraciones como a infecciones oportunistas (Hernández et al., 2018; Patel et al.), en la misma línea se debe promover una buena higiene oral (Sampson et al., 2020; Bao et al., 2020), fundamental para controlar la carga microbiana total en la cavidad oral, manteniendo o restableciendo el equilibrio simbiótico oral, inspirados en estudios metagenómicos que han informado altas lecturas de bacterias cariogénicas y patógenos periodontales en enfermos con COVID19 (Patel et al.; Cox et al.). Los programas de salud deberían mejorar aún más la cobertura en salud oral de la población adulta con morbilidades sistémicas que son muy vulnerables ante situaciones tan extremas como COVID-19 que además enfrentan con malas condiciones de salud oral.

Tal como lo señaló Anaya-Saavedra (2021), en nuestro estudio nos parece que todavía no es posible hablar de manifestaciones de COVID-19 en la Mucosa Oral, más bien hallazgos que acompañan a la enfermedad, asociados al estado crítico de salud de los enfermos. Pero debemos seguir investigando.

Con los antecedentes aportados se propone que la Odontología se debe integrar en los equipos profesionalesque cuidan a enfermos con patologías complejas (Huméres et al., 2020) no solo ante la presencia de infecciones odontogénicas reagudizadas sinode forma preventiva perseverando en educación, con disposición a aportar a la salud del ser humano,sin desestimar la preocupación por la función y la estética, la mirada principal debe ser biológica, la boca es una parte integral de la sa- 
lud de un ser humano, puede reflejar como está realmente la salud del prójimo y en esta enfermedad ya no tan emergente es necesario seguir indagando respecto de señales que contribuyan al conocimiento de la infección por SARS-CoV- 2, COVID-19 sistémicamente y también en la cavidad oral porque son un todo.

\section{AGRADECIMIENTOS}

Nuestros agradecimientos a todo el equipo de profesionales que trabajan a diario con un enorme compromiso humano, ético y profesional para salvar la vida de los enfermos con COVID-19, como asimismo a las autoridades que permitieron el desarrollo de este estudio.

PALACIOS, I. V.; SQUELLA, J. C. \& DOWLING, S. F. Oral health status of a sample of COVID-19 patients from the Dr. Sótero del Río Hospital, Santiago de Chile. Int. J. Odontostomat., 15(4):1026-1034, 2021.

ABSTRACT: In order to know the presence of specific clinical manifestations of COVID-19 in the oral cavity and describe the oral health status of the patients, a basic oral examination was performed on 99 patients with COVID-19 admitted to the Critical Patient Unit (UPC) and in other facilities of the Dr. Sótero del Río Hospital in Santiago during July 2020. Of the total of patients, 51 were in critical condition with mechanical ventilation (IMV), average age 55 years and 48 were without IMV, with 66 years on average, the majority were male, with $25.5 \%$ of women with IMV and $37 \%$ without IMV, Hypertension was the most frequent systemic morbidity among patients followed by Diabetes Mellitus and Obesity both among those in IMV and without it. With regard to oral health, the predominance of ulcerative and erosive lesions was observed in $57 \%$ of people in IMV, however, the number of lesions and their clinical pattern, was nonspecific. The high frequency of dry mouth also stands out, reaching $54 \%$ in critically ill patients and $34 \%$ in less serious ones, the paleness of the mucous membranes and the clinical appearance of the tongue were striking. The presence of periodontal inflammation could be clinically seen, but it was not possible to determine the level of insertion, nor evaluate the patients, with X-rays being only an approximate fact. The teeth were generally in regular state coinciding to what is seen in this population group in Chile. The presence of specific COVID-19 lesions could not be determined in this study group.

KEY WORDS: COVID-19, oral manifestaciones.

\section{REFERENCIAS BIBLIOGRÁFICAS}

Álvarez Lerma, F.; Sánchez García, M.; Lorente, L.; Gordo, F.; Añón, J. M.; Álvarez, J.; Palomar, M.; García, R.; Arias, S.; VázquezCalatayud, M.; et al. Guidelines for the prevention of ventilatorassociated pneumonia and their implementation. The Spanish "Zero-VAP" bundle. Med. Intensiva, 38(4):226-36, 2014.

Amorim Dos Santos, J.; Normando, A. G. C.; Carvalho da Silva, R. L.; Acevedo, A. C.; De Luca Canto, G.; Sugaya, N.; Santos-Silva, A. R. \& Guerra, E. N. S. Oral Manifestations in Patients with COVID-19: A Living Systematic Review. J. Dent. Res., 100(2):141-54, 2021

Anaya-Saavedra, G. Oral manifestations accompanying and related to COVID-19: Overlooking the obvious. Oral Dis., 2021. DOI: https://www.doi.org/10.1111/odi.13857

Baeza, M.; Morales, A.; Cisterna, C.; Cavalla, F.; Jara, G.; Isamitt, Y., Pino, P. \& Gamonal, J. Effect of periodontal treatment in patients with periodontitis and diabetes: systematic review and meta-analysis. J. Appl. Oral Sci., 28:e20190248, 2020.

Bao, L.; Zhang, C.; Dong, J.; Zhao, L.; Li, Y. \& Sun, J. Oral Microbiome and SARS-CoV-2: Beware of Lung Co-infection. Front. Microbiol., $11: 1840,2020$

Biadsee, A.; Biadsee, A.; Kassem, F.; Dagan, O.; Masarwa, S. \& Ormianer, Z. Olfactory and oral manifestations of covid-19: sexrelated symptoms-a potential pathway to early diagnosis. Otolaryngol. Head Neck Surg., 163(4):722-8, 2020.

Bonifaz, A.; Tirado-Sánchez, A.; Paredes-Farrera, F.; Moreno-Moreno, J.; Araiza, J. \& González, G. M. Oral involvement in mucormycosis. A retrospective study of 55 cases. Enf. Infecc. y Microbiol. Clin., 39(10):506-9, 2021.

Brandão, T. B.; Gueiros, L. A.; Melo, T. S.; Prado-Ribeiro, A. C.; Nesrallah, A.; Prado, G.; Santos-Silva, A. R. \& Migliorati, C. A. Oral lesions in patients with SARS-CoV-2 infection: could the oral cavity be a target organ? Oral Surg. Oral Med. Oral Pathol. Oral Radiol., 131(2), e45-e51, 2021.

Coke, C. J.; Davison, B.; Fields, N.; Fletcher, J.; Rollings, J.; Roberson, L.; Challagundla, K. B.; Sampath, C.; Cade, J.; Farmer-Dixon, C.; et al. SARS-CoV-2 Infection and Oral Health: Therapeutic Opportunities and Challenges. J. Clin. Med., 10(1):156, 2021.

Cox, M. J.; Loman, N.; Bogaert, D. \& O'Grady, J. Co-infections: potentially lethal and unexplored in COVID-19. Lancet, 1(1):E11, 2020.

Cruz Tapia, R.; Peraza Labrador, A.; Magno Guimaraes, D. \& Matos Valdez, L. Oral mucosal lesions in patients with SARS-CoV-2 infection. Report of four cases. Are they a true sign of COVID-19 disease? Spec. Care Dent., 40:555-60, 2020.

Egido-Moreno, S.; Valls-Roca, U.; Jané-Salas, E.; López-López, J. \& Estrugo-Devesa, A. COVID-19 and oral lesions, short communication and review. J. Clin. Exp. Dent., 13(3):e287-e294, 2021.

Gupta, S.; Mohindra, R.; Chauhan, P. K.; Singla, V.; Goyal, K.; Sahni, V.; Gaur, R.; Verma, D. K.; Ghosh, A.; Soni, R. K.; et al. SARSCoV-2 Detection in Gingival Crevicular Fluid. J. Dent. Res., 100(2):187-93, 2021.

Hernández Poblete, G. W. La estepa invernal. Santiago de Chile, Ediciones Rumbos, 2000.

Hernandez, G.; Boerma, E. C.; Dubin, A.; Bruhn, A.; Koopmans, M.; Edul, V. K.; Ruiz, C.; Castro, R.; Pozo, M. O.; Pedreros, C.; et al. Severe abnormalities in microvascular perfused vessel density are associated to organ dysfunctions and mortality and can be predicted by hyperlactatemia and norepinephrine requirements in septic shock patients. J. Crit. Care, 28(4):538.e9538.e5.38E14, 2013. 
Hernández, G.; Cavalcanti, A. B.; Ospina-Tascón, G.; Zampieri, F. G.; Dubin, A.; Hurtado, F. J.; Friedman, G.; Castro, R.; Alegría, L.; Cecconi, M.; et al. Early goal-directed therapy using a physiological holistic view: the ANDROMEDA-SHOCK-a randomized controlled trial. Ann. Intensive Care, 8(1):52, 2018.

Hoffmann, M.; Kleine-Weber, H.; Schroeder, S.; Krüger, N.; Herrler, T.; Erichsen, S.; Schiergens, T. S.; Herrler, G.; Wu, N. H.; Nitsche, A.; et al. SARS-CoV-2 Cell Entry Depends on ACE2 and TMPRSS2 and Is Blocked by a Clinically Proven Protease Inhibitor. Cell, 181(2):271-280.e8, 2020.

Hoque, M. N.; Akter, S.; Mishu, I. D.; Islam, M. R..; Rahman, M. S.; Akhter, M.; Islam, I.; Hasan, M. M.; Rahaman, M. M.; Sultana, M.; et al. Microbial co-infections in COVID-19: Associated microbiota and underlying mechanisms of pathogenesis. Microb. Pathog., 156:104941, 2021.

Huméres, C.; Márquez, A.; González-Ritchie, P.; Valenzuela, R.; Rivera, M.; Vásquez, P. \& Araya, C. Multidisciplinary management of the oral cavity in COVID-19 patients under invasive mechanical ventilation. role of the dental team. Int. J. Odontostomat., 14(4):701-4, 2020.

Iranmanesh, B.; Khalili, M.; Amiri, R.; Zartab, H. \& Aflatoonian, M. Oral manifestations of COVID-19 disease: A review article. Dermatol. Ther., 34(1):e14578, 2021.

Jimenez-Cauhe, J.; Ortega-Quijano, D.; Carretero-Barrio, I.; SuarezValle, A.; Saceda-Corralo, D.; Moreno-Garcia Del Real, C. \& Fernandez-Nieto, D. Erythema multiforme-like eruption in patients with COVID-19 infection: clinical and histological findings. Clin. Exp. Dermatol., 45(7):892-5, 2020.

La Rosa, G.; Libra, M.; De Pasquale, R.; Ferlito, S. \& Pedullà, E. Association of Viral Infections With Oral Cavity Lesions: Role of SARS-CoV-2 Infection. Front. Med., 7:571214, 2021.

Machhi, J.; Herskovitz, J.; Senan, A. M.; Dutta, D.; Nath, B.; Oleynikov, M. D.; Blomberg, W. R.; Meigs, D. D.; Hasan, M.; Patel, M.; et al. The natural history, pathobiology, and clinical manifestations of SARS-CoV-2 infections. J. Neuroimmune Pharmacol., 15:359-86, 2020.

Marouf, N.; Cai, W.; Said, K. N.; Daas, H.; Diab, H.; Chinta, V. R.; Hssain, A. A.; Nicolau, B.; Sanz, M. \& Tamimi, F. Association between periodontitis and severity of COVID-19 infection: A casecontrol study. J. Clin. Periodontol., 48(4):483-91, 2021.

Mazucanti, C. H. \& Egan, J. M. SARS-CoV-2 disease severity and diabetes: why the connection and what is to be done?. Immun. Ageing, 17:21, 2020.

Ministerio de Salud. Encuesta Nacional de Salud 2016-2017 Segunda entrega de resultados. Santiago de Chile, Ministerio de Salud, Gobierno de Chile, 2018. Disponible en: https://www.minsal.cl/wpcontent/uploads/2018/01/2-Resultados ENS_MINSAL_31_01_2018.pdf

Ministerio de Salud. Informe Epidemiológico 130 de COVID-19. Santiago de Chile, Ministerio de Salud, Gobierno de Chile, 2020.

Patel, K. P.; Vunnam, S. R.; Patel, P. A.; Krill, K. L.; Korbitz, P. M.; Gallagher, J. P.; Suh, J. E. \& Vunnam, R. R. Transmission of SARSCoV-2: an update of current literature. Eur. J. Clin. Microbiol. Infect. Dis., 39 (11), 2005-2011, 2020.

Sampson, V.; Kamona, N. \& Sampson, A. Could there be a link between oral hygiene and the severity of SARS-CoV-2 infections? Br. Dent. J., 228(12):971-5, 2020.

Sinadinos, A. \& Shelswell, J. Oral ulceration and blistering in patients with COVID-19. Evid. Based Dent., 21(2):49, 2020.

Soares, C. D.; Mosqueda-Taylor, A.; Hernandez-Guerrero, J. C.; de Carvalho, M. \& de Almeida, O. P. Immunohistochemical expression of angiotensin-converting enzyme 2 in minor salivary glands during SARS-CoV-2 infection. J. Med. Virol., 93(4):1905-6, 2021.

Tang, K.; Wang, Y.; Zhang, H.; Zheng, Q.; Fang, R. \& Sun, Q. Cutaneous manifestations of the Coronavirus Disease 2019 (COVID-19): A brief review. Dermatol. Ther., 33:e13528, 2020.
Wang, D.; Hu, B.; Hu, C y col. Clinical Characteristics of 138 Hospitalized Patients With 2019 Novel Coronavirus-Infected Pneumonia in Wuhan, China. JAMA, 323(11):1061-9, 2020.

Dirección para correspondencia:

Dra. Verónica Palacios I.

Facultad de Medicina

Escuela Odontología

Pontificia Universidad Católica de Chile

Patología Oral Servicio de Salud Metropolitano Sur Oriente Santiago

CHILE

Email: vpalaciosi@uc.cl 